\title{
Software Components for Signal Fishing based on GA Element Position Optimizer
}

\author{
N. Crisan, L.C. Cremene, M. Cremene
}

\author{
Nicolae Crisan, Ligia Chira Cremene, \\ Marcel Cremene \\ Technical University of Cluj-Napoca Romania, \\ Cluj-Napoca, Memorandumului nr. 28, 400114 \\ E-mail(s): Nicolae.Crisan@com.utcluj.ro, \\ Ligia.Cremene@com.utcluj.ro, \\ Marcel.Cremene@com.utcluj.ro
}

\begin{abstract}
Long-term adaptation solutions do not receive much attention in the design phase of a wireless system. A new approach is proposed, where the antenna takes an active role in characterising and learning the operation environment. The proposed solution is based on a signal fishing mechanism. Several software components, among which a genetic optimizer, implement the processing stages of autonomous design of the antenna array during operation. Keywords: smart antennas, signal fishing, long-term dynamic adaptation, GA optimizer
\end{abstract}

\section{Introduction}

Wireless receivers incorporate more and more adaptive techniques in the attempt to compensate the effects of the radio channel. Technical challenges for current wireless technologies include limited bandwidth and transmit power, interference, signal fading and other propagation impairments.

A multitude of adaptive techniques are nowadays implemented at different levels of a wireless transmission chain, as depicted in figure 1. At the physical layer, data link and network layers there are many adaptive techniques that deal with modulation and coding schemes, equalization, filtering, ARQ mechanism, fragmentation, routing [9], [10]. These techniques have more or less independent approaches, and their joint operation is not much studied. Adaptive solutions are usually specific solutions, having a local effect. Most of the existing adaptive solutions are hardcoded and therefore not able to evolve.

The main concerns regarding wireless receiver performance are link availability and link capacity. Tens of adaptive techniques are trying to dynamically adapt the transmission to the changes in the wireless environment. These are short-term, instant adaptation methods/mechanisms. Long-term adaptation solutions are more complex and their design and operation are time consuming.

The majority of the adaptation methods considering the spatial component of the propagation channel use highly-parameterized, yet simplified channel models [3], [4]. Usually fixed parameters, such as mean values of statistical channel parameters are involved [5]. Also, processing methods that consider time and frequency variability of the channel exploit only partially the spatial component information.

An approach for developing a long-term dynamic adaptation solution for wireless receiver chains is proposed. Unlike current approaches the antenna takes an active role in characterising and learning the operation environment, namely the wireless propagation channel.

The proposed solution is based on a spatial characterization of the fading allowing the detection of signal maxima in the antenna environment. The antenna is described as an $M x N$ 
rectangular array. Only two elements in the array are active at a given time. In order to search for the best positions of the active elements a genetic optimizer is used.

This paper is organized as follows: Section 2 discuses the interdisciplinary aspect of the smart antenna field. In section 3 we describe several software components that implement the processing stages of the autonomous design of the antenna array during operation. The proposed solution is based on a signal fishing mechanism [6] and uses a genetic optimizer. Experimental results are presented in section 4 together with our conclusions.

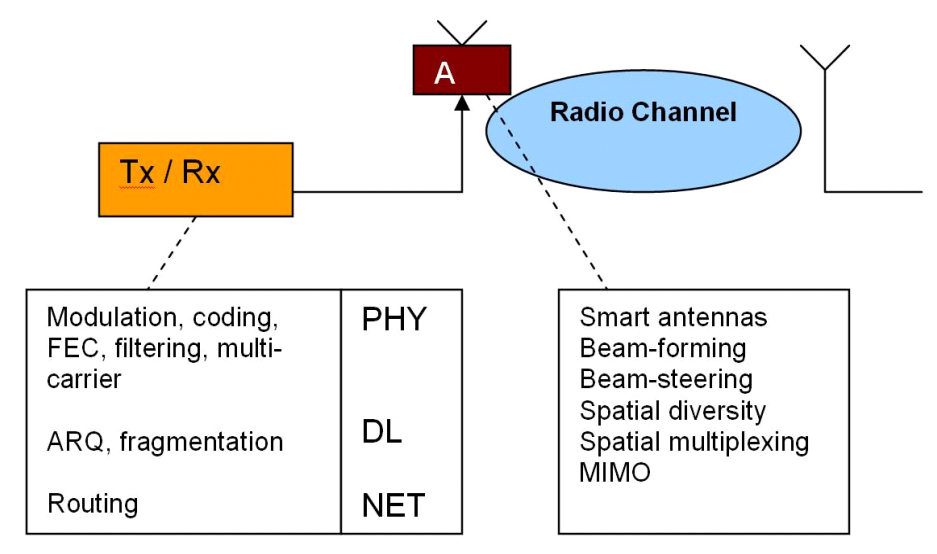

Figure 1: Adaptive techniques operating at different levels of the wireless Tx-Rx chain

\section{Smart antenna interdisciplinary aspect}

Classical smart antennas are based on antenna arrays of different configurations and controlled by means of a specialized signal processor. Their beamforming and beamsteering mechanisms address capacity and reliability issues of wireless systems [14], [15]. The subject of smart antennas, also known as adaptive antennas, becomes a widely interdisciplinary field as it bridges several disciplines: electronics, electromagnetics, channel\&propagation modelling, communication techniques - signal processing, control\&adaptive systems, random processes, and evolutionary computing.

Antenna, communications, and control engineers tend to view adaptive antennas from quite different perspectives. Some are inclined to focus on electromagnetic features (e.g. radiation patterns and levels), others on communication-link parameters like SNR (Signal-to-Noise Ratio) and BER (Bit Error Rate), and others on various control algorithms.

There are at least three issues that computational intelligence (especially evolutionary computing) can help solve in smart antenna systems. The first direction is generating new, optimized antenna geometries, capable of multi-mode operation. The other two are dynamic optimization issues that concern signal detection and tracking and statistical signal processing.

We identify the antenna as a pivotal element in determining and assessing quality in wireless communication systems. Actually, the user-perceived network or equipment quality relies, ultimately, on antenna performance. Moreover, until recently, the antenna influence on channel measurements for channel modelling was considered a bias, now it can be used to a benefit by integrating it into the channel analysis [3], [4].

The distance between transmitter and receiver, their speed, their transmit powers, obstacles, all have an impact on the channel matrix [7], [2], [1]. These are difficult to control factors. Yet, there is a system that has a great influence on the channel matrix, a system that is under the control of the receiver: it is the receiver antenna array. The position of the antenna array in 
the field and the spacing between array elements are the main parameters that can influence the channel matrix.

\section{Proposed solution: Signal fishing based on GA element position optimizer}

Signal fishing (SF) is a new concept [6] that addresses fading mitigation in adaptive multipleantenna receivers. The SF mechanism is based on spatial fading characterization relaying on the antenna array itself. The main idea is to detect and exploit channel signal maxima.

Fading is a random fluctuation of amplitude and phase of the propagated signal. Amplitude fluctuation in a multipath environment is usually described by a Rayleigh distribution. A useful fading characterization will turn the selective-fading channel problem into a flat-fading problem, and will enable the receiver signal processing part to perform better [6].

Antenna selection techniques generally consider a fixed number of elements in the antenna array allowing only regular configurations. Signal fishing allows a random activation of a variable number of array elements which is equivalent to dynamically modifying the element spacing $d$ and the orientation angle $\Omega$.

Figure 2 a) illustrates the description of the receive rectangular array which is part of a transmit-receive system operating in a MIMO $2 \times 2$ configuration. Each element is a Sierpinski gasket (Fig. 2b). Two elements are selected at a specific moment of time and six wave-fronts are considered. The two array elements operate as receive antennas; the other elements are used for channel estimation and spatial fading characterization and are uniformly spaced ( $d$ is constant only for these $\mathrm{Np}$-2 elements). Spatial fading characterization involves estimation or measuring of the angle of arrival $\theta i$, phase shift $\varphi i$, and amplitude $a i$ of the incoming signal which is performed during the training sequence. When the training sequence elapses, the channel is considered quasi-static for about 448 symbols. By changing the element displacement (element perturbation) we can introduce a phase difference between arriving signals $R x 1$ and $R x 2$.
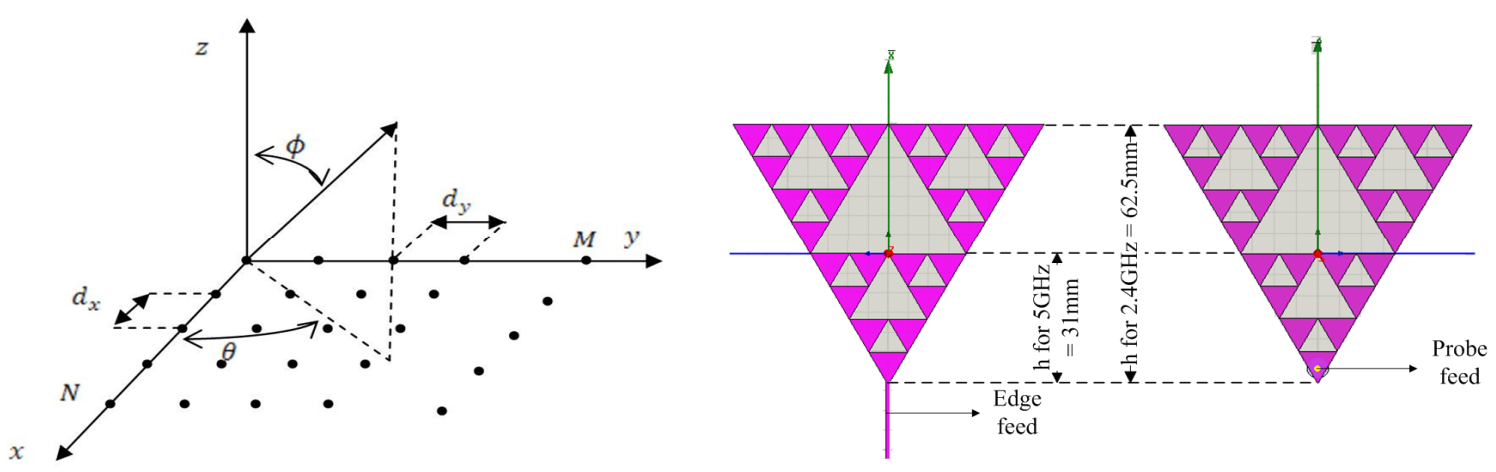

Figure 2: a) 32x32 rectangular antenna array $\phi=90^{0}, 0 \leq \theta \leq 90^{0}, d_{x}=d_{y}=\frac{\lambda}{4}, M=N=32$. b) Sierpinski gasket multi-band antenna

The signal equations for each of the two receive elements, $R x 1$ and $R x 2$, are:

$$
\begin{aligned}
& R_{x 1}(a, b)=\sum_{i=1}^{n_{p}} \rho_{\left(\theta_{i}, 90^{0}\right)} f_{i} \exp \left[j\left((a-1)\left(d_{x} \cos \theta_{i}\right)\right)+j(b-1)\left(d_{y} \sin \theta_{i}\right)\right] \\
& R_{x 2}(c, d)=\sum_{i=1}^{n_{p}} \rho_{\left(\theta_{i}, 90^{0}\right)} f_{i} \exp \left[j\left((c-1)\left(d_{x} \cos \theta_{i}\right)\right)+j(d-1)\left(d_{y} \sin \theta_{i}\right)\right]
\end{aligned}
$$


where: $\rho$ is the antenna element factor

$f_{i}=\left|f_{i}\right| \exp \left(j \phi_{i}\right)$ is the complex value associated to wavefront $i$.

$a, b, c$, and $d$ are the coordinates of the activated receive elements $R x 1$ and $R x 2$

$\beta$ is the phase constant

$\theta i$ is the angle of arrival (AoA) of the $i$ th front.

Figure 3 presents an illustration of the signal fishing concept - exploiting the spatial component information of the channel in order to detect and use the signal maxima. Signal maxima are estimated based on the optimum element spacing

$$
d_{\text {opt }}=\lambda \frac{\sum_{i=1}^{N_{p}}\left|h_{i}\right| \frac{4 K \pm 1}{4 \sin \theta_{i}}}{\sum_{i=1}^{N_{p}}\left|h_{i}\right|}
$$

where $K$ is a positive integer; the array grows wider with $K$.
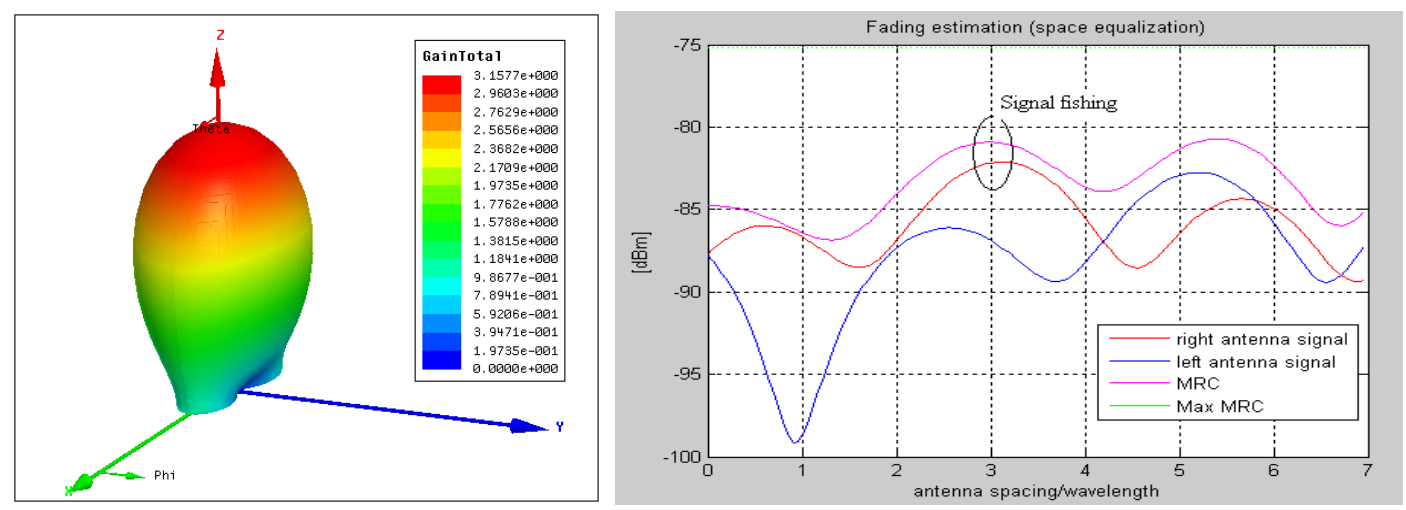

Figure 3: Signal fishing with two antennas

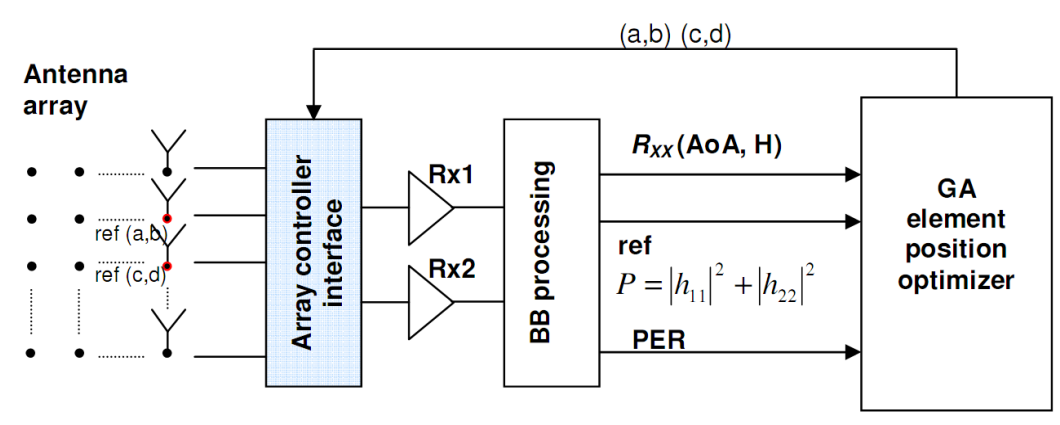

Figure 4: Signal fishing based on GA element position optimizer

Figure 4 shows the block diagram of the signal fishing mechanism realization. The main components are: an $N p$-element antenna array, two receiver chains, an array controller, a baseband processing component and a genetic optimizer.

The array controller plays a key role in the antenna selection mechanism. The array controller can be located between the antenna array and the receiver RF chain. Antenna selection control is based on a switching algorithm that activates two elements at a time. The array controller is the software component that translates the coordinates $a, b, c, d$ into a binary signal suited for on/off element switching. This is done via a microcontroller located on a Software Defined Radio board. This algorithm can be implemented in the context of the SDAA (Software Defined Autonomous Antenna), described by the authors in [11]. 
Benefits of adaptive antenna spacing and angle diversity are discussed in [16]. Passive antenna diversity alone is not enough to ensure significant capacity improvements in MIMO systems. However, spacing and angle reconfiguration of the antenna array is enough to significantly increase the system data rate [8]. Classical digital beamforming and beamsteering algorithms can achieve this but with limited degrees of freedom and introducing noise. Activating the appropriate two array elements results in maximization of $R x 1$ and $R x 2$ signals.

Activating certain elements in the array is equivalent to complex coefficient multiplication (complex weighting) in baseband processing [2]. This spatial method is meant to maximize the SNR by spatial decorrelation of the incoming fronts.

From the adaptive mechanism point of view, the baseband processing component includes two important software components: the PER (Packet Error Rate) calculation component and the $R x x$ correlation matrix estimation component.

The PER component counts the dropped packets and statistically evaluates the packet error rate after thousands of received data packets. This software component is available in most wireless equipments and its value is readable using a software register of the SDR. The PER value is used as a trigger for the genetic search of array element coordinates $a, b, c$ and $d$.

The Rxx component provides the correlation matrix at the receiver; the matrix is not readily available and is computed based on pilot signal information. The $R x x$ correlation matrix of the receiver $(\mathrm{MxM})$ offers the mean power delay profile and power angular profile. The $H$ channel matrix coefficients and angles of arrival (AoA) can be further extracted.

$$
R_{x x}=E\left\{\bar{x}^{-}{ }^{H}\right\}=A R_{S S} A^{H}+R_{n n}
$$

where $E$ is the expectation function and

$-{ }^{H}$ is the Hermitian of the received signal matrix $\bar{x}(M x 1)$

$M$ is the number of elements that are used for power and angle profile estimation

$A$ is the array of the steering vectors $(M x D)$ (one vector for each angle of arrival, $D$ propagation paths are considered and $D<M)$.

$R_{S S}$ is the corellation matrix of the source $(D x D)$ and

$R_{n n}$ is the correlation matrix of the noise $(M x M)$.

Computation of the $H$ matrix is implemented in many wireless standards, such as IEEE $802.11 \mathrm{n}, 802.16 \mathrm{e}$. The $H$ matrix represents the average energy of the channel between receive antenna $j$ and transmit antenna $i$. The angles of arrival are estimated based on the available $R x x$ matrix, using the ESPRIT method [1]. The computation time is not critical in this case because Rxx computation is also a statistical problem. Rxx computation is an ergodic process that depends on the signal-to-noise ratio.

GA element-position optimizer. Genetic Algorithms (GAs) entered the electromagnetic domain in the 1990s, and have been successfully used in antenna design to evolve new antenna geometries [12], [13]. Unlike most approaches, where GAs are involved, our proposal takes into account solution search during device operation. The GA search is based on spatial fading characterisation data.

The signal fishing (SF) adaptive mechanism needs to find the maximum of the mean received signal power $P=\left|h_{11}\right|^{2}+\left|h_{22}\right|^{2}$, after the MRC signal processing. Therefore the SF mechanism problem reduces to the maximization of the objective function $S F:(M x N)^{2}->R^{+}$, where:

$$
\begin{gathered}
S F(a, b, c, d)=\left|R_{x 1}(a, b)\right|^{2}+\left|R_{x 2}(c, d)\right|^{2} \\
S F(a, b, c, d) \rightarrow \max
\end{gathered}
$$


Solution encoding. Candidate solutions are encoded as $x=(a, b, c, d)$, where $a, b, c, d$ are expressed as binary strings.

Fitness assignment. The fitness of solution $x$ is:

$$
\operatorname{eval}(x)=1 / S F(a, b, c, d)
$$

Population model. A generational model where the offspring completely replace the parent population at each generation is considered.

Stopping criterion. The search process stops when the maximum number of generations is reached.

Search operators. Recombination is based on uniform crossover operator. A mask is randomly generated for each pair. One bit mutation is considered.

Parameter setting. Population size - we consider the initial population of 100 chromosomes randomly generated for the first generation. Number of generations - between 100 and 300 generations. Mutation rate -0.01 . The number of mutations is 19 for each generation and is computed [13] with:

$$
\text { nrmut }=\text { mutrate } * n b i t s * n v a r *(n p o p-1)
$$

where: mutrate is the mutation rate $(0,01)$,

nbits is the number of bits/chromosome (5),

nvar is the number of genes for each chromosome $(4-\mathrm{a}, \mathrm{b}, \mathrm{c}, \mathrm{d})$ and

npop is the total population number (100 chromosomes).

nrmut is the number of mutations per generation.

The search process follows the flowchart described in figure 5 .

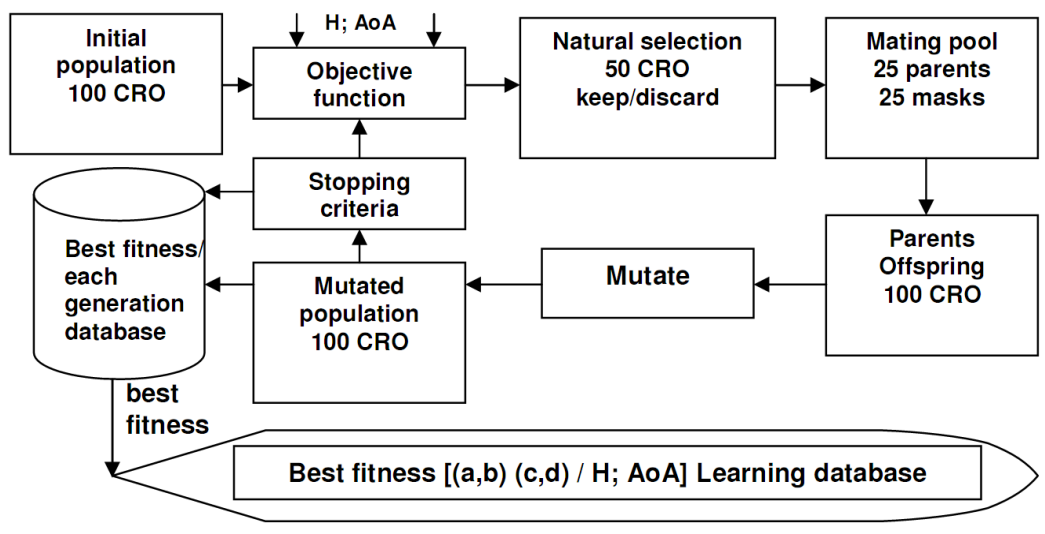

Figure 5: GA optimizer flowchart

\section{Experimental results}

Figure 6 a) illustrates the GA search process. The solution pair is circled in red - Rx1 and $\mathrm{Rx} 2$. It indicates the best coordinates $(a, b)$ for receiving element $R x 1$ and $(c, d)$ for $R x 2$. The corresponding array elements to be activated are shown in figure $6 \mathrm{~b}$ ). The evolution of the objective function for each generation is tracked in Figure 7 a), revealing many local maxima. The global maximum is marked in figure $7 \mathrm{a}$ ). 

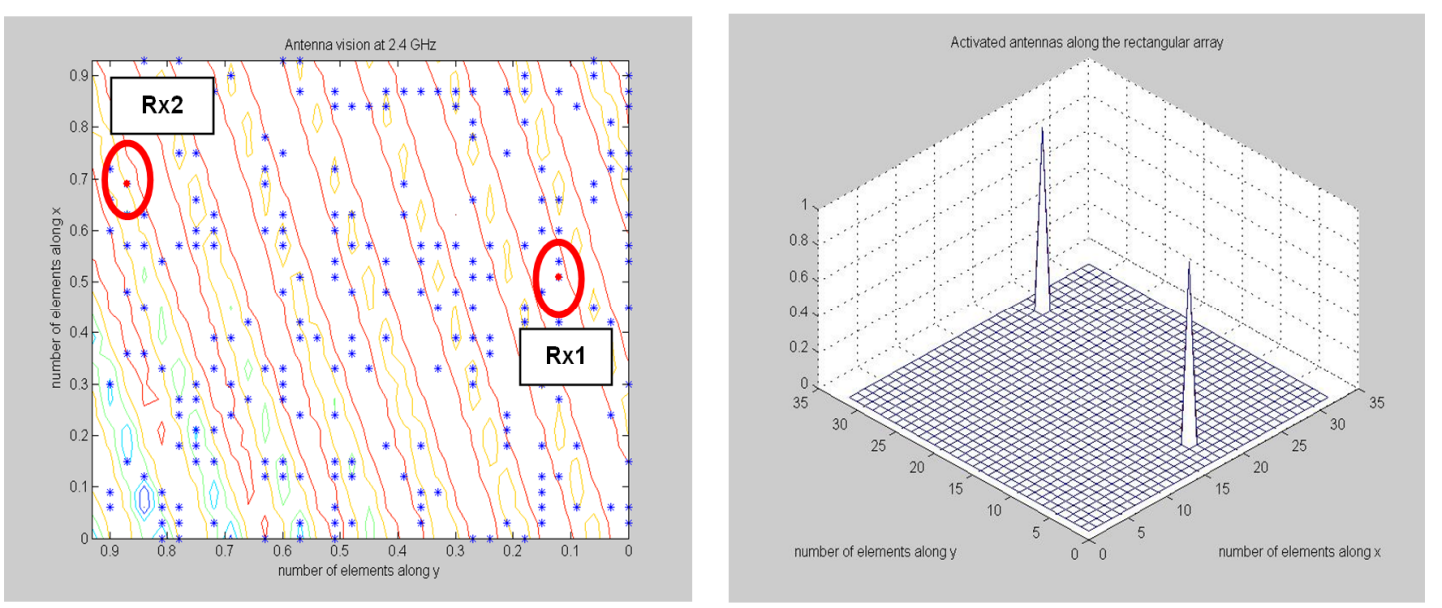

Figure 6: a) GA search process and solution, b) corresponding activated array elements

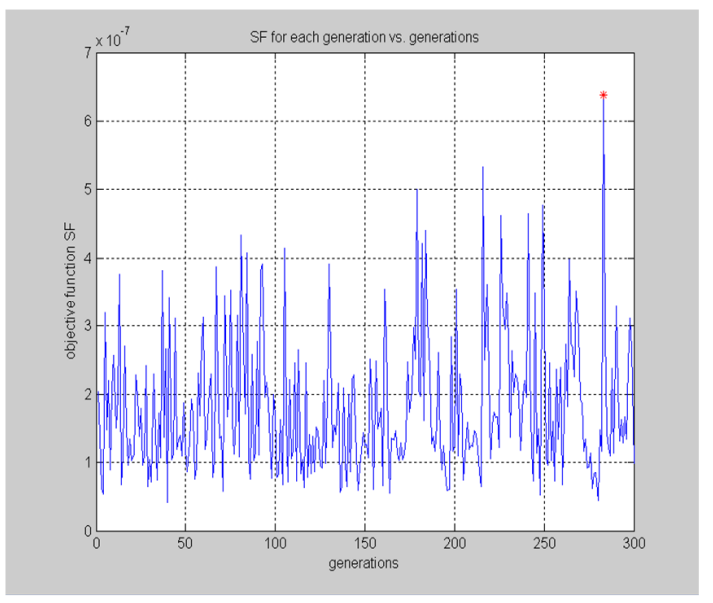

\begin{tabular}{|l|l|l|l|}
$\begin{array}{r}\text { Antenna } \\
\text { tech }\end{array}$ & $\begin{array}{l}\text { Fixed Array } \\
\text { Mean } \\
\text { signal power }\end{array}$ & $\begin{array}{l}\text { Signal Fishing } \\
\text { Mean } \\
\text { signal power }\end{array}$ & $\begin{array}{l}\text { SF } \\
\text { Gain } \\
{[\mathrm{dB}]}\end{array}$ \\
\hline $\begin{array}{l}\text { Direct } \\
\text { search }\end{array}$ & -54.18 & -46.22 & 7.96 \\
\hline GA & -55.31 & -30.94 & 24.37 \\
\hline
\end{tabular}

Figure 7: a) Objective function over generations, b) Signal-fishing mechanism gain 
From the antenna point of view the local maxima are also important because each can result in a signal gain. So whenever a tradeoff between speed and gain is necessary these local maxima can be used. The gain is calculated as the difference between the mean signal power for a fixed two-element array and the mean signal power for the signal fishing mechanism.

\section{Conclusions}

A long-term adaptation solution for wireless receivers is proposed. It is based on a systemic approach of the wireless receiver chain and gives the antenna array a central role in learning the environment, namely the wireless propagation channel. An implementation of the proposed signal-fishing concept using a GA optimizer for element positioning shows that the maximum signal levels of the channel can be detected and used to increase the mean received signal power. The channel fading effects are mitigated starting at the antenna level, an approach that does not introduce additional noise like current baseband processing techniques.

\section{Acknowledgments}

This work was supported by CNCSIS-UEFISCSU, PNII-IDEI, project number 1062/2007.

\section{Bibliography}

[1] F. Gross, Smart antennas for wireless communications, McGraw Hill, 2005

[2] A.B. Gershman, N.D. Sidiropoulos, Space-Time Processing for MIMO Communications, Wiley\&Sons, 2005

[3] 3GPP, "SpatialChannel Model for Multiple Input Multiple Output MIMO simulations," Technical Specification Group Radio Access Network TR 25.996 v6.1.0, 3GPP, Sept. 2003.

[4] J. Salo, G. Del Galdo, J. Salmi, et al., "MATLAB implementation of the 3GPP spatial channel model," Tech. Rep. TR 25.996, 3GPP, Jan. 2005.

[5] L. Mucchi, Claudia Staderini, J. Ylitalo, P. Kyosti, "Modified Spatial Channel Model for MIMO Wireless Systems", EURASIP J. on Wireless Comm. and Netw., vol. 2007, pp.1-7.

[6] N. Crisan, Ligia C. Cremene, "Antenna-based Signal Fishing", The Fifth Int. Conf. on Wireless and Mobile Communications - ICWMC'09, pp.152-156, IEEE Computer Society Press, Cannes, 2009.

[7] G. Tsoulos, MIMO System Technology for Wireless Communications, CRC Press CityplaceBoca Raton, StateFlorida, 2006.

[8] N. Crisan, Ligia C. Cremene, "A Novel Combining Technique for Adaptive Antenna Arrays", ACTA TECHNICA NAPOCENSIS Electronics and Telecommunications, vol. 49/2, pp.27-34, Mediamira Science Publisher, Cluj-Napoca, 2008.

[9] M-S. Alouini, Adaptive and Diversity Techniques for Wireless Digital Communications over Fading Channels, Ph.D. Thesis, California Institute of Technology, 1998.

[10] L. Hanzo, C. H. Wong, M. S. Yee, Adaptive Wireless Transceivers, John Wiley \& Sons, NY, 2002. 
[11] Ligia C. Cremene, N. Crisan, "Towards Cognitive Antenna Systems based on AntennaChannel Co-evolution" 3rd Int. Workshop on Soft Computing Applications - SOFA2009, pp.165-169, Szeged, 2009.

[12] D. Dumitrescu, B. Lazzerini, L. C. Jain, A. Dumitrescu, Evolutionary computation, CRC Press, NY, 2000.

[13] R. L. Haupt, Sue Ellen Haupt, Practical genetic algorithms, John Wiley \& Sons, Inc., 2004.

[14] S. D. Blostein, H. Leib, "Multiple Antenna Systems: Their Role and Impact în Future Wireless Access", IEEE Communications Magazine, vol.41, no.7, pp.94-101, July 2003.

[15] Angeliki Alexiou, M. Haardt, "Smart Antenna Technologies for Future Wireless Systems: Trends and Challenges", IEEE Communications Magazine, vol.42, no.9, pp. 90-97, 2004.

[16] J. D. Boerman, J. T. Bernhard, "Performance Study of Pattern Reconfigurable Antennas in MIMO Communication Systems", IEEE Trans. on Ant. and Propag., Vol.56, No.1, Jan.2008. 